\title{
Survival Benefit of a Monoclonal Antibody against Cadherin-17 in an Orthotopic Liver Tumor Xenograft Model
}

\author{
Kin Tak Chan ${ }^{1}$, Lai Nar Tung ${ }^{1,2}$, Ho Yu Lam ${ }^{1}$, Mei Yuk Choi ${ }^{1}$, Winnie Tan ${ }^{1}$, \\ Mung Yee Wong ${ }^{1,2}$, Sarwat Fatima ${ }^{1,3}$, Pui Ying Tam ${ }^{1}$, Hector K. Wang ${ }^{1}$, \\ Edgar S. L. Liu ${ }^{2}$, Zhao Xiang Bian ${ }^{3}$ and Nikki P. Lee ${ }^{1^{*}}$ \\ ${ }^{1}$ Department of Surgery, The University of Hong Kong, Hong Kong. \\ ${ }^{2}$ College of Life Sciences and Technology, School of Professional and Continuing Education, \\ The University of Hong Kong, Hong Kong. \\ ${ }^{3}$ School of Chinese Medicine, Hong Kong Baptist University, Hong Kong.
}

Authors' contributions

This work was carried out in collaboration between all authors. Authors KTC, LNT, HYL, MYC, WT, MYW, SF, PYT and HKW planned, performed the experiments and analyzed the data. Authors KTC, ESLL, ZXB and NPL designed and commented on the study. Authors KTC and NPL wrote the manuscript. All authors read and approved the final manuscript.

Article Information

DOI: 10.9734/JALSI/2016/22188

Editor(s):

(1) Mohamed A. Jaber, Dubai College of Dental Medicine, MBR University of Medical \& Health Sciences, Dubai, United Arab Emirates. (2) Dongpei Li, Department of Neuroscience and Regenerative Medicine, Institute of Molecular Medicine and Genetics, Georgia Regents University, Augusta, GA 30912, USA.

Reviewers:

(1) Pietro Scicchitano, Murgia Hospital "F. Perinei", Altamura, Italy. (2) Anonymous, Medical University of Lublin, Poland. Complete Peer review History: http://sciencedomain.org/review-history/13498

Original Research Article

Received $22^{\text {nd }}$ September 2015

Accepted $21^{\text {st }}$ November 2015

Published $1^{\text {st }}$ March 2016

\section{ABSTRACT}

Aims: Monoclonal antibodies against tumor-related molecules are therapeutic agents for cancers. Promising results support the use of monoclonal antibodies in several cancers such as lung and breast cancer. However, using monoclonal antibodies as therapeutic agents to treat hepatocellular carcinoma (HCC), a major type of liver cancer, is still at the beginning. We have previously generated a monoclonal antibody against cadherin-17 (CDH17), named Lic5. This antibody has proven anti-tumor and cisplatin-sensitizing effect in HCC using a subcutaneous tumor xenograft mouse model. 
Study Design and Methodology: To further consolidate our previous finding, we tested the effect of Lic5 alone or in combination with cisplatin or epirubicin on tumor growth inhibition and animal survival using a more advanced orthotopic tumor xenograft model.

Results: Single Lic5, cisplatin and epirubicin treatment inhibited growth of cultured HCC cells using cell proliferation assay, while more significant reductions were observed when cisplatin or epirubicin was used together with Lic5. Similar trends of growth inhibition were observed when the same experimental grouping was applied to treat orthotopic tumor-bearing nude mice. Treatment of Lic5 enhanced survivals of orthotopic tumor-bearing nude mice when compared to the control group. Among all experimental groups, combined Lic5 and epirubicin group yielded the best survival. For next phase antibody humanization, we also identified the complementarity determining regions (CDRs) on variable regions on the light and heavy chain of Lic5.

Conclusion: Together, we have validated the preclinical use of Lic5 in an orthotopic HCC xenograft model. Our successful identification of CDRs constitutes the first step in synthesizing humanized Lic5.

Keywords: Cadherin-17; Lic5; cisplatin; epirubicin; survival.

\section{INTRODUCTION}

Global incidence of liver cancer ranks top among various gastrointestinal cancers. More than half a million new cases appear each year, concordant with an approximately equal number of deaths annually. Hepatocellular carcinoma (HCC) is a major type of liver cancer, contributing up to more than $80 \%$ of total cases. Resectable patients can be treated by frontline tumor resection, while such curative treatment does not benefit advanced stage patients presented with multi-nodal or even metastatic tumors. Unlike a number of solid tumors that can be treated quite effectively using chemotherapeutic drugs, HCC is rather resistant to traditional chemotherapeutics including cisplatin and epirubicin. Although molecular targeting drug Sorafenib, a multikinase inhibitor, is clinically approved for treating unresectable, advanced stage patients, its drug efficacy is modest. To improve this clinical situation and to offer new treatment options for patients not amendable for current therapies, it is our longstanding efforts to research for HCCrelated molecules for therapeutic targeting.

Cadherin-17 (CDH17) is an oncofetal molecule first identified by our group to be overexpressed in more than half of the HCC cases [1]. Later studies further demonstrated the clinical correlation of $\mathrm{CDH} 17$ overexpression with advanced HCC condition such as tumor invasion and late stage tumor [2,3]. We deciphered its tumorigenic mechanism by showing its intersection with a frequently deregulated $\mathrm{Wnt} / \beta$ catenin pathway in $\mathrm{HCC}$ that leads to the formation of a novel $\mathrm{CDH} 17 / \beta$-catenin axis. Activation of this axis stimulates the expression of several tumorigenic molecules including cell cycle regulator cyclin D1 [3,4]. Besides, we also unraveled its tumorigenic effects in cultured HCC cells and their derived tumor xenografts in nude mice using a series of functional assays and animal models. These prior studies have put forth the concept of targeting $\mathrm{CDH} 17$ for therapy. Exogenous suppression of $\mathrm{CDH} 17$ using RNA interference or our in-house generated monoclonal antibody against $\mathrm{CDH} 17$ (Lic5) significantly reduced growth of subcutaneous HCC tumors [3,5]. Enhanced suppression on growth of subcutaneous tumors was observed when we combined the use of Lic5 with a conventional chemotherapeutic cisplatin [5]. Our prior results have undoubtedly described the tumor-suppressing effect of Lic5 and its cisplatinsensitizing effect.

Along this research line, this study performed indepth analyses on the tumor-suppressing and chemosensitizing effects of Lic5 on a more clinically relevant orthotopic HCC xenograft model. In addition, we also exploited the complementarity determining regions (CDRs) on the variable regions of heavy chain and light chain of Lic5, for which this information is essential for reducing the immunogenicity of mouse antibody through antibody humanization before clinical application. In all, this study consolidates the preclinical usefulness of Lic5 on HCC by a more advanced animal model. The derived CDR information can lay as a foundation for next phase production of humanized Lic5 for clinical use.

\section{MATERIALS AND METHODS}

\subsection{Human HCC Cell Lines}

Human HCC cell lines MHCC97-L (97L) and $\mathrm{PLC} / \mathrm{PRF} / 5$ (PLC) were used as before $[6,7]$. 
Both cell lines were cultured in Dulbecco's Modified Eagle Medium (DMEM) (Life Technologies, Grand Island, NY, USA) supplemented with $10 \%$ heat-inactivated fetal bovine serum (FBS) (Life Technologies), 100 units $/ \mathrm{ml}$ penicillin and streptomycin (Life Technologies). Cultured cells were maintained in a humidified atmosphere at $3^{\circ} \mathrm{C}$ with $5 \%$ carbon dioxide.

\subsection{Production and Characterization of Lic5, an in-house Monoclonal Antibody against $\mathrm{CDH} 17$}

An in-house hybridoma cell line secreting Lic5 was generated as described [5]. Hybridoma cells were maintained in DMEM supplemented with $10 \%$ FBS and 100 units $/ \mathrm{ml}$ penicillin and streptomycin. To collect Lic5, hybridoma cells were cultured in serum-free hybridoma-SFM (Life Technologies) for 6 days. Cultured supernatant was collected and purified using Protein G column (Life Technologies). Concentration of the purified Lic5 was estimated using RC DC Protein Assay (Bio-Rad, Hercules, CA, USA). Purity of Lic5 was assessed by sodium dodecyl sulfatepolyacrylamide gel electrophoresis (SDS-PAGE) under reducing condition, followed by Coomassie Blue staining. Immunoreactivity and specificity of Lic5 was confirmed by western blot using 97L cell lysate and by immunohistochemistry using paraffin-embedded human HCC tissue. The use of clinical specimen for research was approved by the Institutional Review Board of The University of Hong Kong/Hospital Authority Hong Kong West Cluster (HKU/HA HKW IRB). The procedures for SDS-PAGE, Coomassie Blue staining and western blot were routinely performed [8-10], while the method for immunohistochemistry was described below.

\subsection{In vitro Cell Proliferation Assay}

Pre-determined number of 97L and PLC cells was seeded onto 96-well culture plates for 1 day before treatment with Lic5 and/or chemotherapeutic drugs, cisplatin (Pharmachemie BV, Netherlands) and epirubicin (Pfizer, New York, NY, USA). Cells were subjected to the following treatments for 2 days: (1) $150 \mu \mathrm{g} / \mathrm{ml}$ Lic5; (2) $0.156 \mu \mathrm{g} / \mathrm{ml}$ and 0.625 $\mu \mathrm{g} / \mathrm{ml}$ cisplatin for $97 \mathrm{~L}$ and PLC cells, respectively; (3) $0.04 \mu \mathrm{g} / \mathrm{ml}$ and $0.01 \mu \mathrm{g} / \mathrm{ml}$ epirubicin for 97L and PLC cells, respectively; (4) combined treatment of Lic5 and cisplatin; (5) combined treatment of Lic5 and epirubicin; (6)
$150 \mu \mathrm{g} / \mathrm{ml}$ mouse IgG (Sigma-Aldrich, St Louis, $\mathrm{MO}$, USA) as a control for Lic5. After treatment, proliferation of treated cells was measured using Cell Proliferation Kit I (Roche, Indianapolis, IN, USA) and as described [10]. In brief, MTT reagent was incubated with the treated cells for 4 hours. Colorimetric signals were then measured after addition of $10 \%$ SDS. Independent cell proliferation MTT assays were performed at least twice for result confirmation.

\subsection{Orthotopic Tumor Xenograft Model}

Male nude mice were obtained from Laboratory Animal Unit of The University of Hong Kong, Hong Kong. Animals were housed in individually ventilated cages under a 12-hour light/12-hour dark cycle and with free access to autoclaved water and chow. Animal experiments performed in this study were approved by the Committee on the Use of Live Animals in Teaching and Research (CULATR) of our institute. To allow in vivo viewing of the growing orthotopic liver tumors, previously established luciferase-labelled 97L cell line was used [11]. $5 \times 10^{6}$ luciferaselabelled 97L cells were injected subcutaneously into nude mice to allow the growth of the subcutaneous tumors. These subcutaneous tumors were harvested when their sizes reached 200-300 $\mathrm{mm}^{3}$ and used as tumor seeds to establish orthotopic liver tumors in a separate group of nude mice following the standard procedure [11]. Five days after orthotopic tumor implantation, mice received different treatments as following via intraperitoneal administration 3 times/week for 3 weeks (10-11 mice in each group): (1) $6 \mathrm{mg} / \mathrm{kg} \mathrm{Lic5;} \mathrm{(2)} 1 \mathrm{mg} / \mathrm{kg}$ cisplatin; (3) $1 \mathrm{mg} / \mathrm{kg}$ epirubicin; (4) combined treatment of Lic5 and cisplatin; (5) combined treatment of Lic5 and epirubicin; (6) $6 \mathrm{mg} / \mathrm{kg}$ mouse $\mathrm{lgG}$ as Lic5 control. Every week after treatment, orthotopic tumor growth and metastasis were monitored by live imaging in an IVIS 100 In Vivo Imaging System (Perkin Elmer, Waltham, MA, USA) after intraperitoneal injection of $150 \mathrm{mg} / \mathrm{kg}$ potassium luciferin (Gold Biotechnology, St Louis, MO, USA). For each measurement, a net photon count was obtained for each tumor. At the end of the experiment, orthotopic tumors were collected and subjected for formalin fixation and paraffin embedding.

\subsection{Animal Survival Study}

Orthotopic liver tumors were established as above using 97L cells. Orthotopic tumor-bearing mice received the following treatments 
intraperitoneally 3 times/week for 3 weeks (11-15 mice in each group): (1) $8 \mathrm{mg} / \mathrm{kg}$ Lic5; (2) 1 $\mathrm{mg} / \mathrm{kg}$ cisplatin; (3) $1 \mathrm{mg} / \mathrm{kg}$ epirubicin; (4) combined treatment of Lic5 and cisplatin; (5) combined treatment of Lic5 and epirubicin; (6) 8 $\mathrm{mg} / \mathrm{kg}$ mouse IgG as Lic5 control. Animal survival was continuously monitored till 6 weeks after treatment.

\subsection{Immunohistochemistry}

Immunohistochemistry was performed on paraffin-embedded orthotopic tumor xenografts and human HCC specimen as described [10,12]. In brief, $6 \mu \mathrm{m}$ sections were prepared for deparaffinization and rehydration. After antigen retrieval and hydrogen peroxide treatment, sections were blocked with $3 \%$ bovine serum albumin before staining with mouse monoclonal antibody against $\beta$-catenin (1:200; BD Biosciences, San Jose, CA, USA) and Lic5 $(0.0625 \mu \mathrm{g} / \mathrm{ml})$ overnight at $4^{\circ} \mathrm{C}$. Signals were detected using EnVision+ System-HRP Labelled Polymer Anti-mouse (Dako, Carpinteria, CA, USA) and visualized using Liquid DAB+ Substrate Chromogen System (Dako). Counterstaining was performed using hematoxylin. Images were captured using DXM1200F digital camera (Nikon, Melville, NY, USA).

\subsection{Cloning of Variable Regions of Lic5}

Total RNA was extracted from Lic5-secreting hybridoma cells using TRIzol reagent (Life Technologies) and converted to cDNA using SuperScript III First-Strand Synthesis System (Life Technologies). Variable region cDNA fragments from heavy chain and light chain of Lic5 were amplified using Ig-Primer Set (Novagen, Germany) and Platinum Taq DNA Polymerase High Fidelity (Life Technologies). Variable region of light chain was amplified by the 5' variable region primer (5'ATGGAGACAGACACACTCCTGCTAT-3') and 3' primer on the constant region, while variable region of heavy chain was obtained by the 5 ' variable region primer (5'ATGAACTTYGGGYTSAGMTTGRTTT-3', in which $Y=C / T, S=C / G, M=A / C$, and $R=A / G$ ) and $3^{\prime}$ primer on the constant region. PCR products after resolving on a $1.5 \%$ agarose gel were purified and sequenced (BGI, Hong Kong).

\subsection{Identification of CDR of Lic5}

The nucleotide sequences of variable region of heavy chain and light chain of Lic5 were used to derive the amino acid sequences, which were then numbered according to Kabat numbering scheme. CDR was identified according to Kabat definition (www.bioinf.org.uk).

\subsection{Statistical Analyses}

Statistical analyses were performed using SPSS version 19 (SPSS Inc., Chicago, IL, USA). Data presented in bar chart are expressed as mean \pm $\mathrm{SD} / \mathrm{SEM}$. Student's $t$-test was used to calculate the significance of difference between treatment groups and control group in both cell proliferation MTT assay and animal tumor xenograft experiments. Kaplan-Meier method was employed for survival analyses, while the differences in survival were calculated using logrank test. A $p$-value of less than 0.05 was considered statistically significant.

\section{RESULTS}

\subsection{Establishment of a Pipeline for Purifying Large Quantity of Lic5 from Hybridoma Cell Culture Supernatant}

To obtain Lic5 with high purity and quantity for in vitro and in vivo experiments, we cultured Lic5secreting hybridoma cells in serum-free medium. Our established pipeline for purifying Lic5 achieved a good antibody purity and yield. Coomassie Blue staining result revealed two prominent bands of about 55 and $27 \mathrm{kDa}$ corresponding to the heavy and light chain of antibody on Day 6 culture supernatant, while these bands were not observed on Day 1 culture supernatant. With the presence of detectable level of antibody, culture supernatant collected on Day 6 was subjected to Protein G affinity chromatography for antibody purification. Successful binding of antibody onto the Protein G column was confirmed when we failed to detect the presence of antibody in the flowthrough after loading Day 6 culture supernatant onto the column. Antibody elution performed on different fractions (eluent E1 to E4) revealed the second and third elution fraction contained most abundant antibody. The two stained bands corresponding to the heavy and light chain of the antibody are the predominant bands detected on the protein gel, implicating our antibody purity with more than $90 \%$ (Fig. 1A). Using our antibody purification pipeline, we managed to collect a favorable antibody amount of $23.88 \pm 6.96 \mathrm{mg}$ Lic5 from each liter of culture supernatant collected on Day 6. 
The purified Lic5 demonstrated high immunoreactivity against $\mathrm{CDH} 17$. A single band of about $120 \mathrm{kDa}$ corresponding to the apparent molecular weight of $\mathrm{CDH} 17$ was detected in CDH17-expressing 97L cells when we used Lic5 as a primary antibody for western blot (Fig. 1B). When Lic5 was used for immunohistochemistry to detect $\mathrm{CDH} 17$ in human HCC tissue, discrete stains appeared at the cell membrane and cytoplasm (Fig. 1C). The immunohistochemical stains generated by using Lic5 in this study was similar to the previous report with the use of a commercial antibody against $\mathrm{CDH} 17$ for immunohistochemistry [1].

\subsection{Survival Benefit of Lic5}

Our previous report has demonstrated the chemosensitizing effect of Lic5 towards a commonly used chemotherapeutic drug cisplatin in a subcutaneous tumor xenograft model [5]. To consolidate our earlier finding, we examined the chemosensitizing effect of Lic5 towards another chemotherapeutic drug epirubicin, which is also commonly used in HCC. Besides, we performed the drug testing with or without Lic5 in a more clinically relevant orthotopic tumor xenograft model.

Before treating orthotopic tumor-bearing mice with the purified Lic5, we first examined the antitumor effect of Lic5 alone or combined with cisplatin or epirubicin in cultured HCC cell lines in vitro using cell proliferation MTT assay (Fig. 2A). When we performed cell proliferation assay on cultured $97 \mathrm{~L}$ cells, treatment with cisplatin and epirubicin for 2 days resulted in $31.6 \%$ and $21.9 \%$ growth inhibition, respectively. Combination with Lic5 further enhanced the growth inhibition effect by 1.74 -fold for cisplatin and 1.98-fold for epirubicin, which led to a growth inhibition of $55.1 \%$ and $43.3 \%$, respectively (Fig. 2A, left panel). Similar growth inhibition was observed in cultured PLC cells, in which treatment with cisplatin resulted in $37.6 \%$ growth inhibition and combined treatment with Lic5 further increased the growth inhibition by 1.73fold $(65.2 \%)$. Unlike the significant growth inhibition of epirubicin on cultured 97L cells, this drug only inhibited growth of cultured PLC cells by $8.1 \%$. Likewise, its combination with Lic5 enhanced the growth inhibition by 4.62 -fold and led to a significant inhibition of $37.4 \%$ (Fig. 2, right panel).

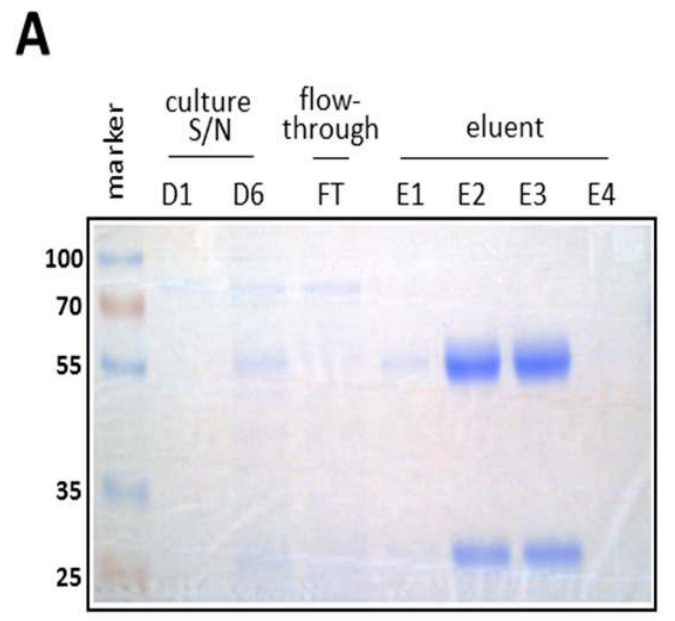

B

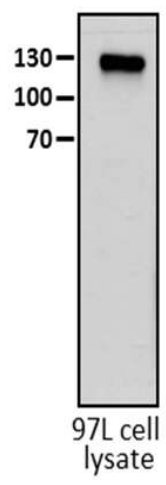

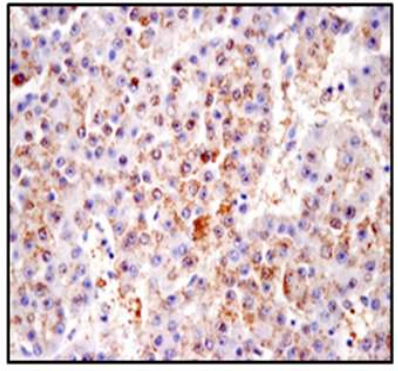

Human HCC tissue

Fig. 1. Purification of Lic5 and its characterization

Lic5 was purified using Protein G affinity chromatography from culture supernatant of Lic5-secreting hybridoma cell line. (A) As shown on the Coomassie Blue-stained gel, culture supernatant (culture S/N) from Day 6 (D6) contained higher antibody amount than Day 1 (D1). Two stained bands with sizes of about $55 \mathrm{kDa}$ and $27 \mathrm{kDa}$ corresponding to the heavy chain and light chain of antibody were visualized on the gel. No antibody was detected in the flow-through (FT) after we passed the Day 6 culture supernatant on the Protein G column.

Antibody elution was performed on 4 fractions (E1 to E4). Most abundant antibody was eluted in fractions E2 and E3, which was collected and pooled. (B) Using purified Lic5 for western blot on 97L cell lysate, a single band corresponding to the apparent molecule weight of $\mathrm{CDH} 17$ was revealed. (C) Using purified Lic5 for immunohistochemistry on human HCC tissue, discrete stains corresponding to the membrane and cytoplasmic localizations of $\mathrm{CDH} 17$ were detected, for which this staining pattern corresponded well to our published report [1] 
A
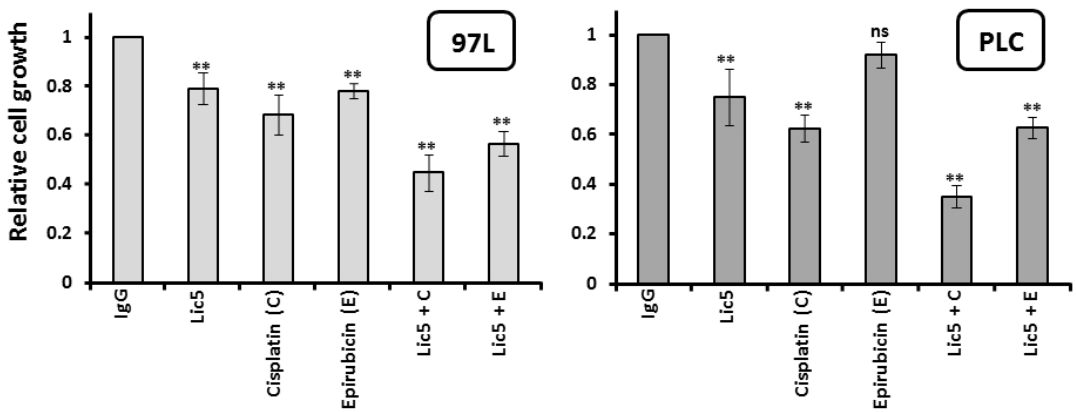

B

\section{C}
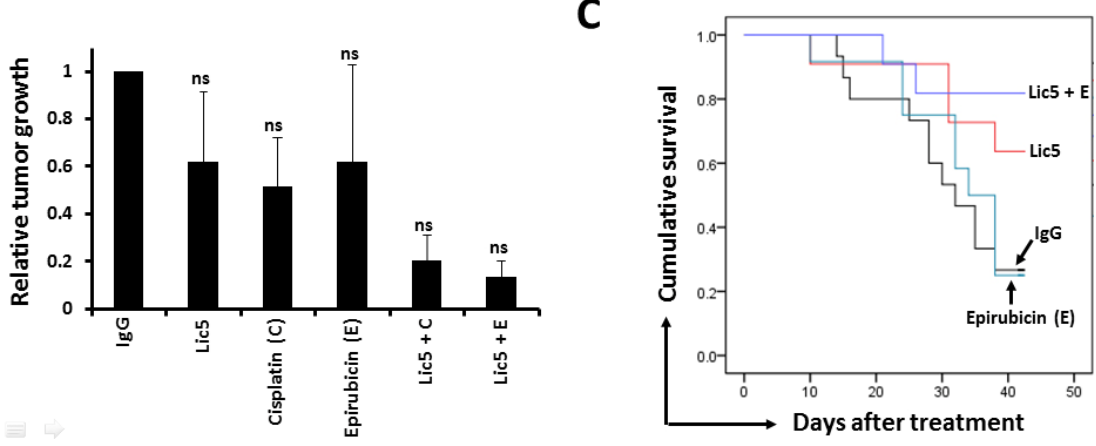

Fig. 2. Chemosensitizing effect of Lic5

(A) Single treatment of Lic5, cisplatin or epirubicin for 2 days inhibited the growth of cultured $97 \mathrm{~L}$ and PLC cells when compared to the mouse IgG-treated cells in cell proliferation MTT assay.

Further growth inhibition was observed when we treated the cultured cells under combined treatment of Lic5 with cisplatin or epirubicin. Bars represent mean $\pm S D$. (B) Luciferase-labelled $97 \mathrm{~L}$ cells were used to establish orthotopic tumor xenografts in nude mice. Single and combined treatments of Lic5 with or without chemotherapeutic drugs cisplatin and epirubicin were applied to these animals for 3 weeks. The data shown are net photon count relative to the control lgG group obtained 3 weeks after treatment. Single treatment yielded tumor growth inhibition, while further reduction on tumor growth was observed when Lic5 was combined with cisplatin or epirubicin. Bars represent mean + SEM. (C) Orthotopic tumor xenografts were established in nude mice using $97 \mathrm{~L}$ cells. These animals were treated with single or combined treatment of Lic5 and epirubicin. Combined treatment of Lic5 and epirubicin led to most favorable survival as revealed in the Kaplan-Meier curve.

A statistical significant difference in survivals was reached when we compared the combined treatment group with mouse IgG control group ( $p=0.017$ ). Abbreviations used: $C$, cisplatin; $E$, epirubicin. ${ }^{* *}, p<0.05$; $n s$, statistically not significant

After confirming the chemosensitizing effect of Lic5 towards cisplatin and epirubicin treatment on cultured HCC cells by cell proliferation MTT assay, we examined whether similar growth inhibition was observed in an in vivo setting by animal tumor xenograft experiments. We used luciferase-labelled $97 \mathrm{~L}$ cells to develop orthotopic tumor xenografts for treatments as this enables live monitoring of tumor growth. When compared to IgG control group, cisplatin treatment inhibited the xenograft growth by $48.6 \%$. Such treatment when combined with Lic5 further augmented the xenograft growth inhibition by 1.64 -fold to $79.6 \%$. Despite such inhibition, the combined treatment data did not yield a statistical significant value when compared to control group $(p=0.08)$. For epirubicin, its combined treatment with Lic5 manifested a further reduction of xenograft growth from $37.9 \%$ for single epirubicin treatment to $86.4 \%$ (i.e. 2.28 -fold). However, such drop in xenograft growth still did not reach a statistical significant value $(p=0.07)$ (Fig. $2 \mathrm{~B})$. At the end of the experiment, we collected the orthotopic tumors for analyzing the expression and localization of $\beta$-catenin, which is known as a downstream molecule of $\mathrm{CDH} 17$ and is a key member of the Wnt signaling pathway. Both combined treatment of Lic5 with cisplatin or epirubicin reduced the overall expression and cytoplasmic localization of $\beta$-catenin when compared to single treatment of chemotherapeutic drugs (Supplementary Fig. 1). Such immunohistochemical results further strengthen the tumor-suppressing effect mediated by Lic5 via its action on altering the expression and localization of $\beta$-catenin. 
As shown in Fig. 2B, a trend of tumor growth reduction was observed for every treatment group when compared to control group. We then compared the survival rate of orthotopic tumorbearing mice for each treatment group versus control group. The animal survival rate of Lic5 group reached $63.6 \%$, which was much higher than that of the control group (26.6\%). Although the survival rate between control group and epirubicin group was similar (26.6\% versus $25 \%$ ), combined treatment of Lic5 and epirubicin led to the most significant increase in survival rate to $81 \%$ among all experimental groups when compared to control group ( $p=0.017$ ) (Fig 2C). However, such significant difference in survival for cisplatin group and cisplatin with Lic5 group did not reach any statistical significant values when compared to control group (data not shown). Taken together, this set of animal experiments has demonstrated the chemosensitizing effect of Lic5 in particular for epirubicin on tumor growth inhibition and survival rate induction.

\subsection{Identification of CDR on Variable Regions of Lic5}

Each monoclonal antibody has its unique variable regions on heavy chain and light chain, for which each of them is composed of a sequence of amino acids that determines the antigen binding property of this antibody. To obtain the variable region sequences from heavy chain and light chain of Lic5, we amplified each variable region using discrete primer pairs (Fig. 3, left panel). After Sanger sequencing of the amplified products, the nucleotide sequences were translated into amino acid sequences by in silico method. Kabat numbering scheme and CDR definition were employed to identify the three CDRs on the variable regions of heavy chain and light chain of Lic5 using the deduced amino acid sequences (Fig. 3, right panel).

\section{DISCUSSION}

This study continues our previous study on testing the anti-tumor and chemosensitizing effect of Lic5 on HCC. Our previous study examined such properties of Lic5 in a subcutaneous tumor xenograft model [5], while this current study adopted the use of a more clinically relevant orthotopic tumor xenograft model. Although subcutaneous xenograft model is widely used for preclinical drug screening, the use of orthotopic xenograft model associates with added advantage by growing the tumor xenograft in the same environment as in the clinical situation. Therefore, orthotopic xenograft model is a more advanced animal model for preclinical drug testing, which can be used effectively for monitoring target site drug delivery, drug efficacy and pharmacokinetics [13]. Despite such advantage, establishment of orthotopic xenograft is more technically demanding due to the need to pre-label implanted tumor seeds for

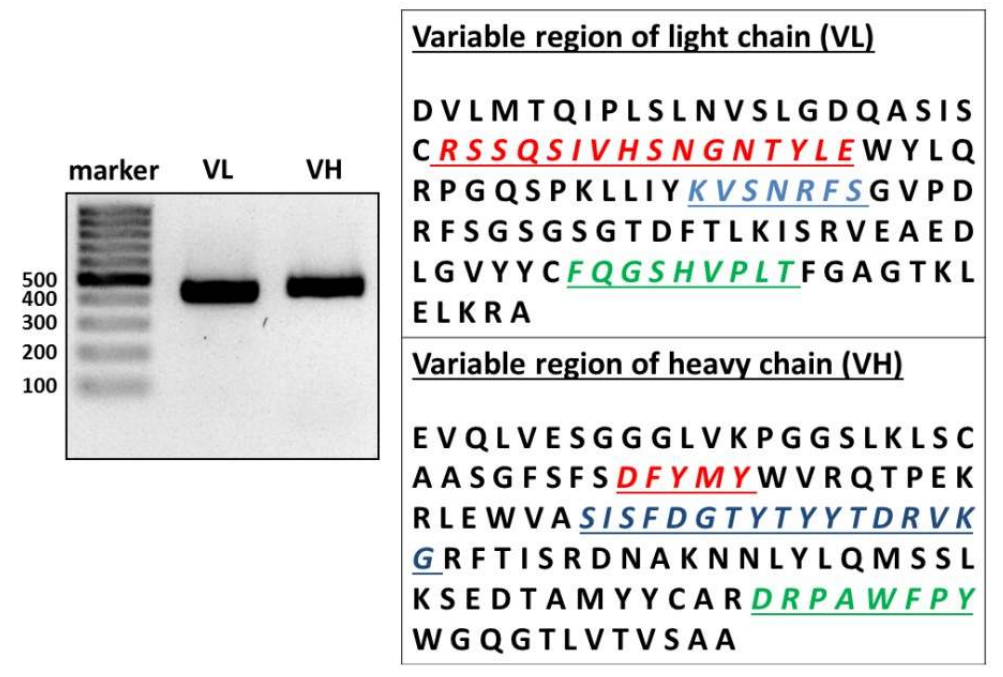

Fig. 3. Amino acid sequences of the variable regions of light chain and heavy chain of Lic5 PCR followed by Sanger sequencing were used to obtain the variable region sequences of light chain and heavy chain of Lic5 from cDNA prepared from Lic5-secreting hybridoma cells (left panel). Based on the Kabat numbering scheme and CDR definition, the six CDRs on the two variable regions were identified and highlighted in different colors (right panel) 
real-time monitoring of xenograft growth and the need for animal surgery for tumor seed implantation. Till now, it is still unsure whether tumor seed pre-labelling would introduce unpredicted factor that might interfere the drug efficacy assessment. Even though we observed a drop in tumor growth of the luciferase-labelled orthotopic tumors after Lic5 treatment, this growth inhibition did not reach a statistical significant difference when compared to the $\lg G$ treatment. This experimental result did not match well with our previous result showing a significant growth inhibition of non-labelled subcutaneous tumors after treating with Lic5 [5]. Similar result deviation was noticed when we compared the use of luciferase-labelled orthotopic tumors with non-labelled subcutaneous tumors under combined treatment of Lic5 with cisplatin.

Our results have demonstrated the anti-tumor and chemosensitizing effects of Lic5 in treating HCC xenografts, implicating our in-house generated monoclonal antibody might be useful to treat other cancers apart from HCC. Lic5 was a monoclonal antibody produced in parallel with Lic3 by our team earlier using recombinant ectodomain 1-2 of CDH17 as an immunogen [3]. Since Lic5 targets the extracellular region of $\mathrm{CDH} 17$, it can bind to intact form or carboxylterminal truncated form of $\mathrm{CDH} 17$ when used for therapeutic purpose. Therefore, it is obvious Lic5 can be used to target other cancers with $\mathrm{CDH} 17$ overexpression. In addition to HCC, a number of cancers are found overexpressing $\mathrm{CDH} 17$, which include gastric adenocarcinoma, esophageal adenocarcinoma, and colorectal cancers [4,14]. Among these cancer types, $\mathrm{CDH} 17$ overexpression was present in majority of colorectal and esophageal adenocarcinoma cancers [15,16]. As such, these $\mathrm{CDH} 17-$ overexpressing cancers might also be susceptible to the action of Lic5. Several studies performing $\mathrm{CDH} 17$ suppression counteracted tumorigenesis as manifested by reduced growth of cultured cells or tumor xenografts after treatment $[17,18]$. Thus, targeting $\mathrm{CDH} 17$ to counteract tumorigenesis seems to work well in other cancers overexpressing $\mathrm{CDH} 17$ in addition to HCC. This opens up an opportunity of targeting $\mathrm{CDH} 17$ using Lic5 in other cancers for therapy.

In this and our previous studies, we have reported targeting $\mathrm{CDH} 17$ using Lic5 inactivated $\mathrm{CDH} 17 / \beta$-catenin axis by interfering with the expression and localization of the axis components such as $\beta$-catenin [5]. Except for one study on gastric cancer that demonstrated similar result [19], no other studies have yet reported this linkage between $\mathrm{CDH} 17$ and $\beta$ catenin-associated network. Instead in another cancer type, CDH17 was bridged to integrinrelated pathway during colorectal tumorigenesis because of the intrinsic integrin-binding affinity of $\mathrm{CDH} 17$ [20,21]. CDH17 also affects two other tumorigenic pathways related to Ras/Raf/MEK/ERK and NFKB, such that its suppression inactivated these pathways in gastric cancer $[22,23]$. Given these observations, it remains to be determined whether Lic5 could also block these pathways as a mean to suppress tumorigenesis.

Apart from testing the anti-tumor effects of Lic5, we also identified the three CDRs on each variable region on heavy and light chains of Lic5, for which these CDRs are responsible for determining antibody binding affinity and specificity [24]. As mouse antibody can elicit detrimental immunogenic responses in humans due to the human anti-mouse antibody reaction, humanization of Lic5, which is a mouse monoclonal antibody, is a prerequisite if we plan to use this antibody in humans in the future. With our identified CDRs on Lic5, next phase will involve generation of humanized Lic5 by grafting the identified CDRs and related amino acid sequence to a human antibody framework. The methodology for antibody humanization has evolved throughout these decades and a number of traditional and updated methods are developed for antibody humanization [25]. All these methods serve the same purpose by removing as much mouse sequences on antibody as possible, while at the same time retaining the essential CDR sequences. Other properties including stability and pharmacokinetics also need careful optimization during the process of antibody humanization [26]. For Lic5 with proven anti-tumor and chemosensitizing effects, we will perform antibody humanization. Upon completion, humanized Lic5 will then undergo extensive and vigorous testing before it can be considered for use in humans.

\section{CONCLUSION}

This paper examines the potential use of a monoclonal antibody Lic5 in treating $\mathrm{HCC}$ xenografts in nude mice. Results derived from this study have validated the preclinical use of Lic5 and envisioned the potential use of Lic5 as HCC therapy. 


\section{FUNDING SUPPORT}

This study was supported by Innovation and Technology Support Programme (Tier 3) (ITS/280/11) of Innovation and Technology Commission and Seed Funding Programme for Applied Research of The University of Hong Kong.

\section{COMPETING INTERESTS}

Authors have declared that no competing interests exist.

\section{REFERENCES}

1. Wong BW, Luk JM, Ng IO, Hu MY, Liu KD, Fan ST. Identification of liver-intestine cadherin in hepatocellular carcinoma--a potential disease marker. Biochem Biophys Res Commun. 2003;311:618-24.

2. Ding ZB, Shi YH, Zhou J, Shi GM, Ke AW, Qiu SJ, Wang XY, Dai Z, Xu Y, Fan J. Liver-intestine cadherin predicts microvascular invasion and poor prognosis of hepatitis B virus-positive hepatocellular carcinoma. Cancer. 2009;115:4753-65.

3. Liu LX, Lee NP, Chan VW, Xue W, Zender L, Zhang $\mathrm{C}$, Mao M, Dai $\mathrm{H}$, Wang $\mathrm{XL}, \mathrm{Xu}$ $\mathrm{MZ}$, Lee TK, Ng IO, Chen Y, Kung HF, Lowe SW, Poon RT, Wang JH, Luk JM. Targeting cadherin-17 inactivates Wnt signaling and inhibits tumor growth in liver carcinoma. Hepatology. 2009;50:1453-63.

4. Lee NP, Poon RT, Shek FH, Ng IO, Luk JM. Role of cadherin-17 in oncogenesis and potential therapeutic implications in hepatocellular carcinoma. Biochim Biophys Acta. 2010;1806:138-45.

5. Wang $Y$, Shek FH, Wong KF, Liu LX, Zhang $X Q$, Yuan $Y$, Khin E, Hu MY, Wang $\mathrm{JH}$, Poon RT, Hong W, Lee NP, Luk JM. Anti-cadherin-17 antibody modulates betacatenin signaling and tumorigenecity of hepatocellular carcinoma. PLoS One. 2013;8:e72386.

6. Au V, Tsang FH, Man K, Fan ST, Poon RT, Lee NP. Expression of ankyrin repeat and SOCS box containing 4 (ASB4) confers migration and invasion properties of hepatocellular carcinoma cells. Biosci Trends. 2014;8:101-10.

7. Tsang FH, Au V, Lu WJ, Shek FH, Liu AM, Luk JM, Fan ST, Poon RT, Lee NP. Prognostic marker microRNA-125b inhibits tumorigenic properties of hepatocellular carcinoma cells via suppressing tumorigenic molecule elF5A2. Dig Dis Sci. 2014;59:2477-87.

8. Lee NP, Tsang $S$, Cheng $\mathrm{RH}$, Luk JM. Increased solubility of integrin bA domain using maltose-binding protein as a fusion tag. Protein Pept Lett. 2006;13:431-5.

9. Lee NP, Leung KW, Cheung N, Lam BY, Xu MZ, Sham PC, Lau GK, Poon RT, Fan ST, Luk JM. Comparative proteomic analysis of mouse livers from embryo to adult reveals an association with progression of hepatocellular carcinoma. Proteomics. 2008;8:2136-49.

10. Fatima S, Lee NP, Tsang FH, Kolligs FT, $\mathrm{Ng}$ IO, Poon RT, Fan ST, Luk JM. Dickkopf 4 (DKK4) acts on Wnt/b-catenin pathway via influencing b-catenin in hepatocellular carcinoma. Oncogene. 2012;31:4233-44.

11. Ng KT, Xu A, Cheng Q, Guo DY, Lim ZX, Sun CK, Fung JH, Poon RT, Fan ST, Lo $\mathrm{CM}$, Man K. Clinical relevance and therapeutic potential of angiopoietin-like protein 4 in hepatocellular carcinoma. Mol Cancer. 2014;13:196.

12. Lee NP, Leung KW, Wo JY, Tam PC, Yeung WS, Luk JM. Blockage of testicular connexins induced apoptosis in rat seminiferous epithelium. Apoptosis. 2006; 11:1215-29.

13. Ruggeri BA, Camp F, Miknyoczki S. Animal models of disease: Pre-clinical animal models of cancer and their applications and utility in drug discovery. Biochem Pharmacol. 2014;87:150-61.

14. Takamura M, Yamagiwa S, Matsuda $\mathrm{Y}$, Ichida T, Aoyagi Y. Involvement of liverintestine cadherin in cancer progression. Med Mol Morphol. 2013;46:1-7.

15. Panarelli NC, Yantiss RK, Yeh MM, Liu Y, Chen YT. Tissue-specific cadherin $\mathrm{CDH} 17$ is a useful marker of gastrointestinal adenocarcinomas with higher sensitivity than CDX2. Am J Clin Pathol. 2012; 138:211-22.

16. Su MC, Yuan $R H$, Lin $C Y$, Jeng $Y M$. Cadherin-17 is a useful diagnostic marker for adenocarcinomas of the digestive system. Mod Pathol. 2008;21:1379-86.

17. Liu QS, Zhang J, Liu M, Dong WG. Lentiviral-mediated miRNA against liverintestine cadherin suppresses tumor growth and invasiveness of human gastric cancer. Cancer Sci. 2010;101:1807-12.

18. Zhang J, Liu QS, Dong WG. Blockade of proliferation and migration of gastric 
cancer via targeting $\mathrm{CDH} 17$ with an artificial microRNA. Med Oncol. 2011;28: 494-501.

19. Quu HB, Zhang LY, Ren C, Zeng ZL, Wu WJ, Luo HY, Zhou ZW, Xu RH. Targeting CDH17 suppresses tumor progression in gastric cancer by downregulating Wnt/bcatenin signaling. PLoS One. 2013;8: e56959.

20. Bartolome RA, Pelaez-Garcia A, Gomez I, Torres S, Fernandez-Acenero MJ, Escudero-Paniagua B, Imbaud JI, Casal Jl. An RGD motif present in cadherin 17 induces integrin activation and tumor growth. J Biol Chem. 2014;289:34801-14.

21. Bartolome RA, Barderas R, Torres S, Fernandez-Acenero MJ, Mendes $M$, Garcia-Foncillas J, Lopez-Lucendo M, Casal Jl. Cadherin-17 interacts with a2b1 integrin to regulate cell proliferation and adhesion in colorectal cancer cells causing liver metastasis. Oncogene. 2014;33:165869.
22. Lin Z, Zhang C, Zhang M, Xu D, Fang Y, Zhou Z, Chen $X$, Qin $N$, Zhang $X$. Targeting cadherin-17 inactivates Ras/Raf/MEK/ERK signaling and inhibits cell proliferation in gastric cancer. PLoS One. 2014;9:e85296.

23. Wang J, Kang WM, Yu JC, Liu YQ, Meng QB, Cao ZJ. Cadherin-17 induces tumorigenesis and lymphatic metastasis in gastric cancer through activation of NFkB signaling pathway. Cancer Biol Ther. 2013;14:262-70.

24. Schroeder HW Jr, Cavacini L. Structure and function of immunoglobulins. J Allergy Clin Immunol. 2010;125:S41-52.

25. Safdari $Y$, Farajnia S, Asgharzadeh $M$, Khalili M. Antibody humanization methods A review and update. Biotechnol Genet Eng Rev. 2013;29:175-86.

26. Kuramochi T, Igawa $\mathrm{T}$, Tsunoda $\mathrm{H}$, Hattori K. Humanization and simultaneous optimization of monoclonal antibody. Methods Mol Biol. 2014;1060:123-37.

(0) 2016 Chan et al.; This is an Open Access article distributed under the terms of the Creative Commons Attribution License (http://creativecommons.org/licenses/by/4.0), which permits unrestricted use, distribution, and reproduction in any medium, provided the original work is properly cited.

Peer-review history:

The peer review history for this paper can be accessed here: http://sciencedomain.org/review-history/13498 